\title{
Cybersecurity Aspects of Heavy Vehicle Digital Forensics
}

\author{
Jeremy S. Daily, Ph.D., P.E. \\ Department of Mechanical Engineering \\ The University of Tulsa \\ 800 S. Tucker Dr. \\ Tulsa, OK 74104 \\ jeremy-daily@utulsa.edu
}

\begin{abstract}
As vehicles become more computerized and cybersecurity concerns transcend traditional information technologies, new challenges related to incident response and digital forensics arise for heavy vehicles. The presentation is focused on highlighting observations related to the cybersecurity of heavy vehicles from the perspective of a mechanical engineer reconstructing traffic crashes. This intersection of cyber-physical incident response will only grow in importance with the race towards autonomy and higher levels of computerized control over the vehicles. As autonomous vehicles are involved in crashes, digital forensics and physical reconstructions will go together to determine crash causation. However, experts in cybersecurity and digital forensics rarely work with experts in traffic crash reconstruction. This presentation tries to bridge that domain gap by providing interesting results from tests and research that would benefit both domains.
\end{abstract}

\section{CCS Concepts/ACM Classifiers}

- Applied computing Data recovery • Applied computing Investigation techniques

\section{Author Keywords}

Heavy vehicle cybersecurity, digital forensics, J1939, incident response, event data recorders

\section{BIOGRAPHY}

After some time working in electronic maintenance in the U.S. Air Force, Jeremy Daily graduated with his Ph.D. from Wright State University after studying mechanical engineering. He went to work at the University of Tulsa researching traffic crash reconstruction techniques where he started a crash reconstruction research consortium (TU-CRRC). As part of that consortium, Dr. Daily traveled all over the United States and performed crash tests to gather data relating digital information onboard vehicles with the physical observations and measurements from a crash scene. Around 2010, Dr. Daily started a US Department of Justice project with colleagues from the computer science department (John Hale and Mauricio Papa). The project was to develop digital forensic techniques for the process control systems onboard heavy trucks. This research resulted in a patent that was awarded in 2018. After the patent application was submitted, Dr. Daily took a sabbatical and founded Synercon Technologies in 2013 with some of his graduating students. After raising venture capital to build digital forensic tools for heavy trucks, the business turned profitable in a couple years and Synercon Technologies was sold in December of 2018. Meanwhile, the heavy vehicle industry recognized the need to understand cybersecurity concerns for their vehicles. In 2017, Dr. Daily co-founded (with Karl Heimer) the CyberTruck Challenge, a non-profit dedicated to creating a community of interest regarding heavy vehicle cybersecurity issues and helping develop the next generation of talent to face these issues. Currently, Dr. Daily teaches in the mechanical engineering department and conducts externally funded research related to heavy vehicle cybersecurity at the University of Tulsa.

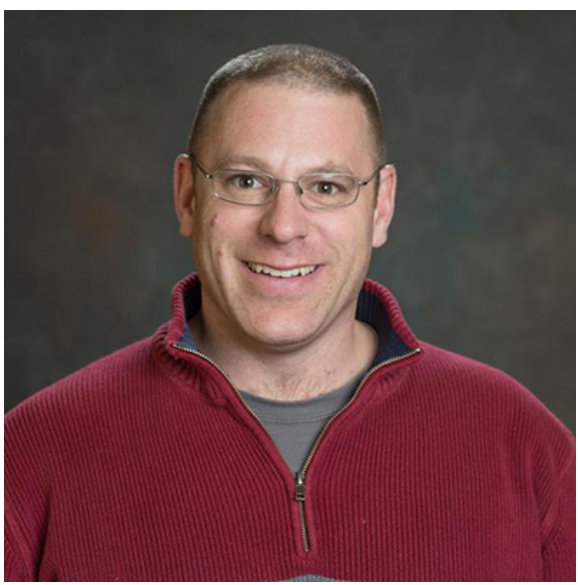

